Voland, Katja; Westendarp, Andreas

Positionspapier 'Beton ohne Steinkohlenflugasche' Veränderung der Betonbauweise aufgrund abnehmender Verfügbarkeit von Steinkohlenflugasche und anderer Kraftwerksnebenprodukte als Zusatzstoffe für Beton

Originalveröffentlichung / Original Publication:

https://doi.org/10.1002/best.202100014

Verfügbar unter / Available at:

https://hdl.handle.net/20.500.11970/108352

Vorgeschlagene Zitierweise / Suggested citation:

Voland, Katja; Westendarp, Andreas (2021): Positionspapier 'Beton ohne Steinkohlenflugasche' Veränderung der Betonbauweise aufgrund abnehmender Verfügbarkeit von Steinkohlenflugasche und anderer Kraftwerksnebenprodukte als Zusatzstoffe für Beton. In: Beton- und Stahlbetonbau 116 (4). S. 322-325. https://doi.org/10.1002/best.202100014.

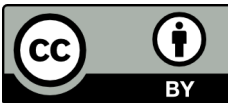




\section{Positionspapier „Beton ohne Steinkohlenflugasche“}

\section{Veränderung der Betonbauweise aufgrund abnehmender Verfügbarkeit von Steinkohlenflugasche und anderen Kraftwerksnebenprodukten als Zusatzstoffe für Beton}

\begin{abstract}
Aufgrund des schrittweisen Ausstiegs aus der Kohleverstromung stehen bereits heute deutlich geringere Mengen an in Deutschland produzierten Steinkohlenflugaschen zur Verfügung. Spätestens im Jahr 2038 wird ihr Anteil auf null fallen. Der Deutsche Beton- und Bautechnik-Verein E.V. (DBV) sowie die Bundesanstalt für Wasserbau (BAW) verfolgen mit diesem Positionspapier das Ziel, alle am Bau Beteiligten über die daraus resultierenden Lieferengpässe von Steinkohlenflugasche zu informieren und Verständnis dafür zu schaffen. Zudem zeigen sie in diesem Papier erste Lösungsansätze auf, um die negativen Auswirkungen auf die Betonbauweise weitestgehend zu minimieren.
\end{abstract}

Stichworte Beton; Steinkohlenflugasche; Lieferengpässe; Positionspapier; Kohleausstieg

\section{1}

Hintergrund

Als ein Element der Energiewende hat der Deutsche Bundestag am 3. Juli 2020 beschlossen, bis zum Jahr 2038 schrittweise aus der Kohleverstromung auszusteigen. Dies wirkt sich unmittelbar auf die Menge der in Deutschland zur Verfügung stehenden Steinkohlenflugaschen aus. Während vor wenigen Jahren noch ca. 3,1 Mio. Tonnen Steinkohlenflugaschen zur Verfügung standen, werden im Jahr 2020 voraussichtlich nur noch 2,1 Mio. Tonnen anfallen, Bild 1, [1]. Aufgrund der derzeitigen COVID-19-Pandemie ist sogar ein noch deutlicherer Abfall zu erwarten. Die Nachfrage nach Strom aus Steinkohle ist zudem u. a. jahreszeitlich bedingt und in Abhängigkeit vom Angebot von Strom aus erneuerbaren Energien starken Schwankungen unterworfen.

Infolge dieser Situation muss davon ausgegangen werden, dass Steinkohlenflugaschen künftig zumindest temporär nicht mehr jederzeit und flächendeckend uneingeschränkt verfügbar sein werden. Detailliertere Informationen können dem Anhang entnommen werden.

\section{Ziel dieses Positionspapiers}

Ziel dieses Positionspapiers ist es, alle am Bau Beteiligten über die Lieferengpässe von Steinkohlenflugasche zu informieren, Verständnis dafür zu schaffen und erste Lösungsansätze aufzuzeigen. Insbesondere soll dahinge-
Position paper "Concrete without hard coal fly ash" - Change in concrete construction due to decreasing availability of hard coal fly ash and other power plant by-products as additives for concrete

Due to the gradual phase-out of coal-fired power generation, significantly lower volumes of hard coal fly ash produced in Germany are already available today. By 2038 at the latest, their share will fall to zero. The German Society for Concrete and Construction Technology (DBV) and the Federal Waterways Engineering and Research Institute (BAW) are pursuing the aim of this position paper to inform all those involved in construction about the resulting supply bottlenecks of hard coal fly ash and to create understanding. In addition, in this paper they show initial approaches to solutions to minimize the negative effects on concrete construction as far as possible.

Keywords concrete; hard coal fly ash; supply bottlenecks; position paper; Fossil-fuel phase-out

hend sensibilisiert werden, dass nicht erst die Bauausführenden mit der zunehmenden Nichtverfügbarkeit von Steinkohlenflugasche und deren Auswirkungen konfrontiert werden. Vielmehr sollte dieser Umstand bereits bei der Planung und Bemessung berücksichtigt werden.

\section{3}

\section{Problemstellung}

Die signifikante Verknappung und bereits heute vorhandenen lokalen Lieferengpässe von Steinkohlenflugaschen sind ein großes und kontinuierlich wachsendes Problem für die Betonbauweise. Und auch wenn Steinkohlenflugaschen anfallen, ergeben sich zum Teil enorme Qualitätsschwankungen. In manchen Fällen entsprechen Steinkohlenflugaschen nicht (mehr) den in Deutschland bestehenden Anforderungen an Steinkohlenflugaschen für die Herstellung von Beton [2] und können daher nicht für die Betonherstellung verwendet werden.

Steinkohlenflugaschen werden bei vielen Bauteilen aus betontechnologischen Gründen als Betonzusatzstoff eingesetzt, um eine angemessene Dauerhaftigkeit des Betons zu erzielen. Dazu gehört z. B. ein ausreichender Widerstand gegenüber einer Alkali-Kieselsäure-Reaktion, einem Sulfatangriff und dem Eindringen von Chloriden von außen in den Beton. Darüber hinaus sind nach derzeitigem Stand insbesondere in Ingenieur- und Infrastrukturbauwerken sowie allgemein in massigen Bauteilen Betone mit Steinkohlenflugasche kaum verzichtbar [3]. Durch 
den Ersatz von Steinkohlenflugasche kann Zement substituiert, die entstehende Hydratationswärme damit reduziert und eine Beanspruchung durch frühen Zwang verringert werden. Zusammengefasst ist festzustellen, dass nach derzeitigem Kenntnisstand in vielen Fällen auf den Einsatz von Steinkohlenflugasche aktuell nur schwer verzichtet werden kann.

Die kontinuierlich zunehmende Steinkohlenflugascheverknappung und die daraus resultierenden Lieferengpässe für die Betonindustrie sind zumindest auf nationaler Ebene aufgrund der klimapolitischen Ziele und der daraus resultierenden Gesetzgebung in Form des Kohleausstiegsgesetzes nicht vermeidbar. Kommunizieren die Betonhersteller die Nichtverfügbarkeit von Steinkohlenflugasche sehr kurzfristig oder gar nicht, kann dies weitreichende Folgen für alle am Bau Beteiligten haben. Im schlimmsten Fall kann das Bau-Soll durch den Bauausführenden nicht mehr erfüllt werden. Kostensteigerungen und Bauzeitverlängerungen sind durch zusätzliche Planungen und Prüfungen in diesem Zusammenhang unvermeidbar. So kann z. B. eine komplett neue Erstprüfung des Betons mit entsprechend hohem Zeitaufwand erforderlich werden. Darüber hinaus kann die regional und jahreszeitlich stark schwankende Verfügbarkeit von Steinkohlenflugasche $\mathrm{zu}$ variierenden Frisch- und auch Festbetoneigenschaften führen.

\section{4}

\section{Lösungsansätze}

Um negative Auswirkungen auf die Betonbauweise möglichst gering zu halten, gibt es verschiedene Möglichkeiten, die verfolgt werden können. Dabei sollten sowohl vergabe- und bauvertragsrechtliche als auch betontechnologische und bautechnische Ansätze berücksichtigt werden. Ferner ist es ggf. erforderlich, die Regelwerkssetzung anzupassen.

Nach derzeitigem Kenntnisstand sollten zunächst insbesondere die folgenden Ansätze verfolgt werden:

1. Die Verfügbarkeit von Steinkohlenflugasche sollte stets bereits bei der Planung und Bemessung des Betonbauteils/Betonbauwerks berücksichtigt werden.

a. Sofern möglich, sollte bei der Planung und Bemessung ein Beton ohne Steinkohlenflugasche gewählt werden. Dies ist insbesondere bei länger andauernden Baumaßnahmen von großer Bedeutung.

b. Bei der Planung und Bemessung mit Steinkohlenflugasche sollte hingegen sichergestellt werden, dass Steinkohlenflugasche während des gesamten Bauablaufs auch tatsächlich zur Verfügung steht. Sofern möglich, empfiehlt es sich z.B., entsprechende Kontingente zu sichern. In diesem Zusammenhang ist jedoch darauf hinzuweisen, dass ein gewisses Restrisiko kaum vermeidbar ist. Um dieses Risiko möglichst gering zu halten, sollten Vorgehensweisen mit und ohne Steinkohlenflugasche verfolgt werden. Insbesondere sollten Alternativ- rezepturen ohne Steinkohlenflugasche vorgehalten werden.

2. Um Bauabläufe möglichst zielsicher planen zu können, sollte eine verbesserte und frühzeitige Kommunikation zwischen allen am Bau Beteiligten erfolgen. Dieser Forderung wird z.B. durch den Entwurf des BBQ-Konzepts [4-7] Rechnung getragen.

3. Prinzipiell sollte Steinkohlenflugasche zukünftig bei eingeschränkter Verfügbarkeit zielorientiert eingesetzt werden.

\section{5}

\section{Ausblick}

Aufgrund des Kohleausstiegsgesetzes wird Steinkohlenflugasche stetig abnehmend für die Herstellung von Beton zur Verfügung stehen und voraussichtlich im Jahr 2034 (vgl. Bild 1) zumindest als Produkt aus deutschen Kraftwerken komplett wegfallen. Das Angebot und die Qualität an ausländischen Steinkohlenflugaschen sind aktuell nicht ausreichend, um den Bedarf zu decken. Inwieweit Hüttensandmehl als Alternative zur Steinkohlenflugasche künftig in ausreichender Menge verfügbar sein wird, ist vor dem Hintergrund langfristiger struktureller Veränderungen in der Eisen- und Stahlherstellung nicht absehbar, derzeit aber auch nicht anzunehmen. Eine erhöhte Nachfrage nach anderen Primärrohstoffen oder Zementhauptbestandteilen, wie z. B. Kalksteinmehl, Trass und kalzinierte Tone, mit denen zumindest teilweise Langzeiterfahrungen im Betonbau bestehen, wird die Folge sein.

\section{Anhang}

Um die weitere Erwärmung des Klimas zu bremsen, hat sich Deutschland das Ziel gesetzt, bis zum Jahr 2050 eine weitgehend treibhausgasneutrale Wirtschaft und Gesellschaft zu erreichen. Die Energieversorgung und der Ausstieg aus der Kohleverstromung nehmen in diesem $\mathrm{Zu}$ sammenhang eine große Bedeutung ein. Im Jahre 2018 setzte die Bundesregierung hierfür die Bundeskommission „Wachstum, Strukturwandel und Beschäftigung“ (WSB-Kommission) ein. Sie sollte unter Berücksichtigung von Klimaschutz, Wirtschaftswachstum und Arbeitsplatzerhalt Empfehlungen für den Ausstieg aus der Kohleverstromung erarbeiten. Im Februar 2019 legte die WSBKommission ihren Abschlussbericht [8] vor. Darin schlug sie vor, die Kohleverstromung schrittweise bis zum Jahre 2038 auslaufen zu lassen. Bereits im Jahr 2020 sollen die ersten vier Gigawatt Steinkohlekraftwerke abgeschaltet werden. Geplant ist, dass im Jahr 2022 nur noch Kraftwerke mit einer Leistung von insgesamt 15 Gigawatt am Netz sind. Bis zum Jahr 2030 soll die Leistung der Steinkohlekraftwerke weiter schrittweise auf nur noch acht Gigawatt reduziert werden. Bereits im Jahr 2033 soll das letzte Steinkohlekraftwerk vom Netz gehen, Bild 1. Am 3. Juli 2020 hat der Deutsche Bundestag nunmehr den schrittweisen Kohleausstieg bis 2038 beschlossen. Der Kohleausstieg wirkt sich unmittelbar auf die Menge der in 


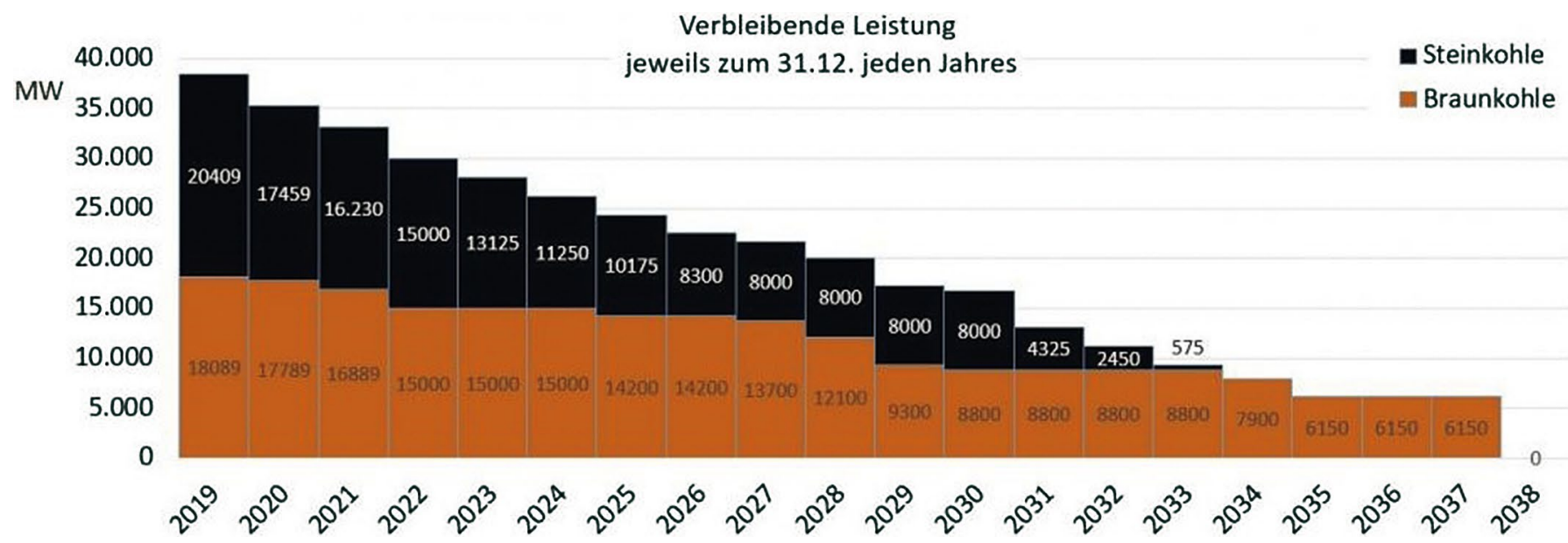

Bild 1 Geplanter Ausstiegspfad für die Braun- und Steinkohle [9]

Planned phase-out path for lignite and hard coal [9]

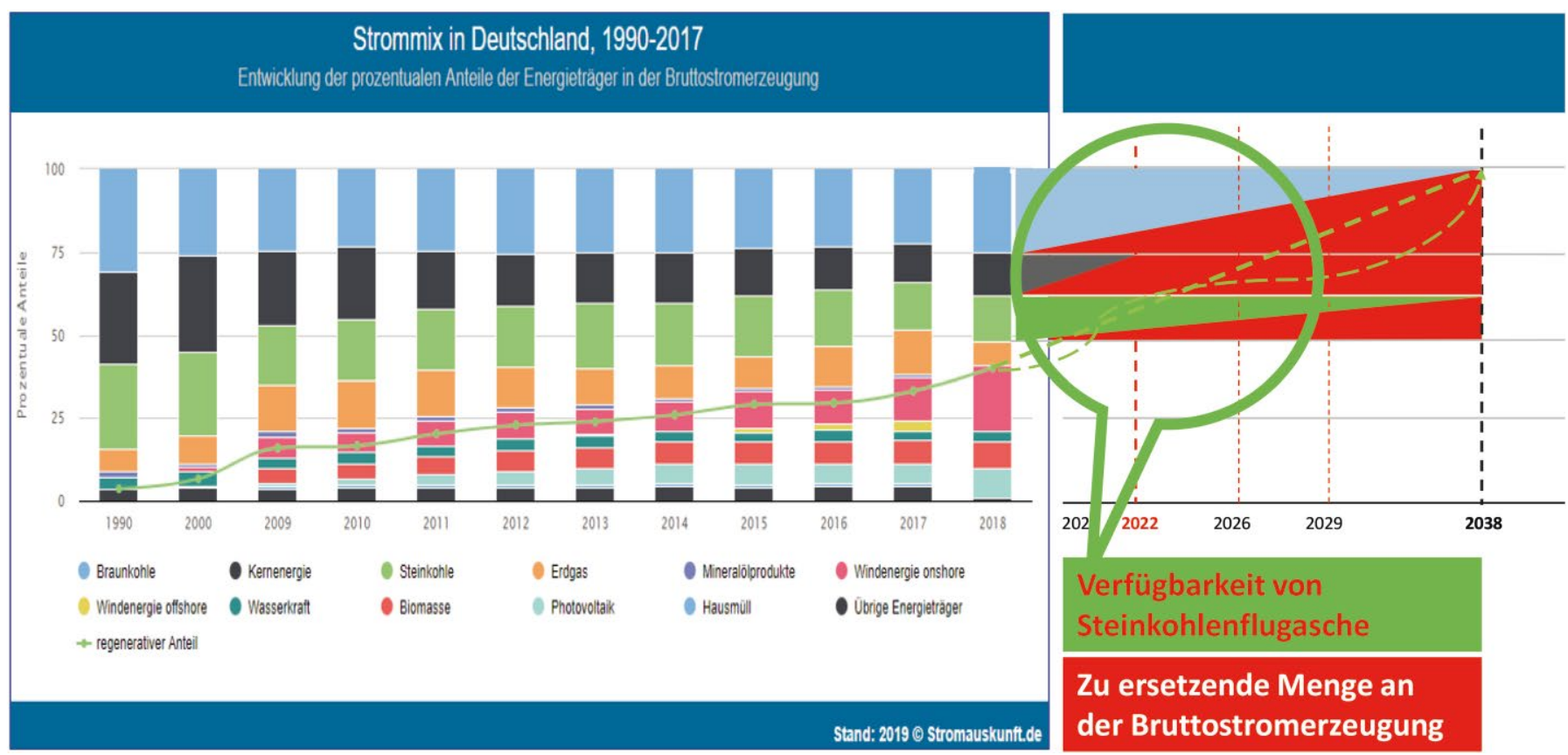

Bild 2 Entwicklung der prozentualen Anteile der Energieträger in der Bruttostromerzeugung von 1990 bis 2018 [10] Development of the percentage shares of energy sources in gross electricity generation from 1990 to 2018 [10]

Deutschland zur Verfügung stehenden Kraftwerksnebenprodukte aus. Neben REA-Gips ist insbesondere Steinkohlenflugasche als Zusatzstoff für Beton betroffen. Wie unter Abschn. 3 Problemstellung beschrieben, hat dies einen immensen Einfluss auf die Betonbauweise.

Nicht nur der Kohleausstieg, sondern auch weitere Faktoren führen dazu, dass Steinkohlenflugasche nicht kontinuierlich verfügbar sowie deren Verfügbarkeit regional und jahreszeitlich stark schwankend ist. Der Hauptfaktor hierfür liegt in der Abschaltung von Kraftwerken aufgrund von Überstrom durch erneuerbare Energiequellen, wie z. B. Energie aus Sonne, Wind, Wasser, Biomasse etc. Wie Bild 2 verdeutlicht, nimmt der prozentuale Anteil der Gesamtenergie aus erneuerbaren Energiequellen aufgrund der deutschen Klimapolitik seit Jahren kontinuierlich zu. Darüber hinaus sind aktuell noch einige Atomkraftwerke in Betrieb. Ferner sind ungeplante und längere Revisionsvorgänge (mit einer Dauer von bis zu acht
Wochen) einzelner deutscher Kraftwerke nicht vermeidbar. All diese Faktoren führen dazu, dass Lieferengpässe und Ausfallszenarien bei Steinkohlenflugasche zum Regelfall werden.

Wie Bild 3 (links) zeigt, war die Produktionsmenge an Steinkohlenflugasche im vergangenen Jahrzehnt mit Werten von 3,9 bis 4,5 Mio. Tonnen auf einem recht hohen Niveau. Von 2010 bis 2016 konnten im Vergleich dazu nur noch Mengen von ca. 3,1 Mio. Tonnen Steinkohlenflugasche erzielt werden. Seitdem sinkt das Aufkommen an Steinkohlenflugasche kontinuierlich. Basierend auf Berechnungen nach Empfehlungen der WSB-Kommission werden 2020 voraussichtlich nur noch 2,1 Mio. Tonnen produziert werden. Durch die derzeitige Covid19-Pandemie ist sogar ein deutlich drastischer Abfall zu erwarten.

Bei vollständigem Kohleausstieg wird spätestens im Jahr 2038 der Wert an in Deutschland produzierten Flug- 

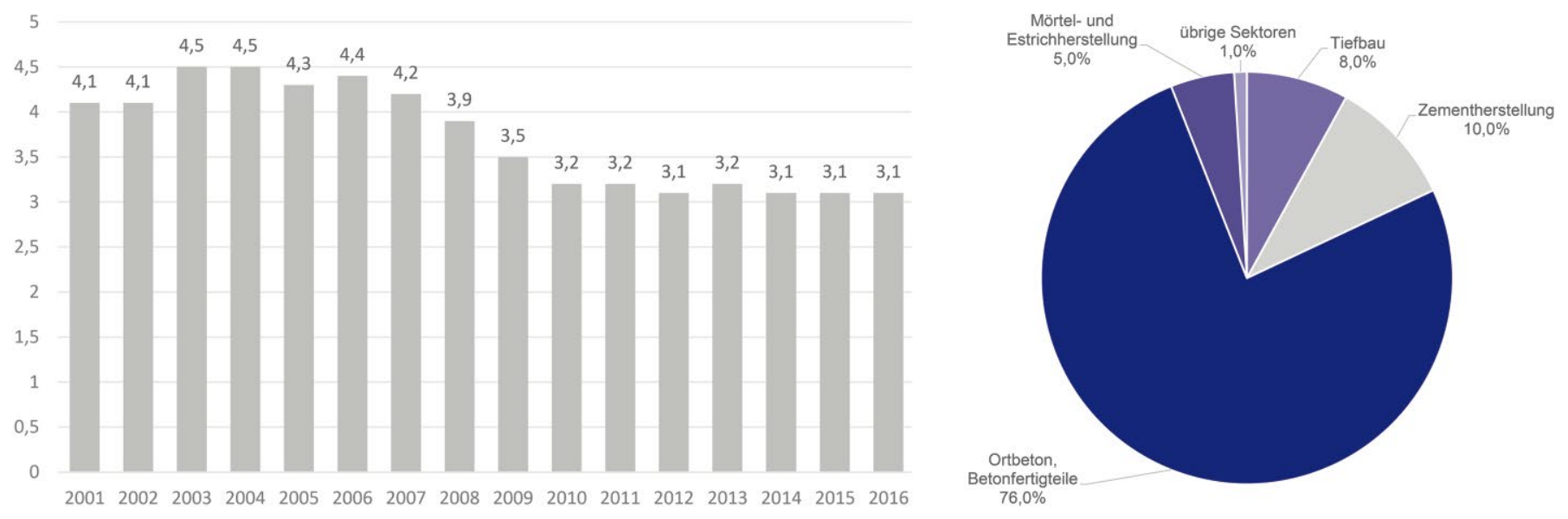

Bild 3 Produktionsmenge an Steinkohlenflugasche in Deutschland bis 2016 zzgl. Berechnung der zukünftigen Produktionsmengen nach Empfehlungen der WSB-Kommission (links) und deren Verwendung in Deutschland im Jahr 2016 (rechts) [11]

Production volume of hard coal fly ash in Germany until 2016 plus calculation of future production volumes according to the recommendations of the WSB Commission (left) and its use in Germany in 2016 (right) [11]

aschen auf null fallen. Diese Entwicklung hat einen zunehmenden Import zertifizierter Steinkohlenflugaschen aus Polen, Tschechien, Benelux etc. zur Folge. Der Hauptteil der im Jahr 2016 produzierten Steinkohlenflugaschen $(76 \%)$ wurde als Zusatzstoff zur Herstellung von Ortbeton und Betonbauteilen eingesetzt (vgl. Bild 3 rechts). 10\% kamen bei der Zementherstellung als Sekundärrohstoff oder Hauptbestandteil zur Anwendung. Die übrigen Anteile verteilten sich auf den Tiefbau (8\%), die Mörtel- und Estrichherstellung (5\%) und übrige Sektoren $(1 \%)$.

\section{Literatur}

[1] Studie Die Nachfrage nach Primär- und Sekundärrohstoffen der Steine-und-Erden-Industrie bis 2035 in Deutschland, Bundesverband Baustoffe - Steine und Erden e.V., 2019 mit Verweis auf VGB PowerTech e.V.

[2] DAfStb-Richtlinie Anforderungen an Ausgangsstoffe zur Herstellung von Beton nach DIN EN 206-1 in Verbindung mit DIN 1045-2 (Ausgabe 8/2019), http://www.dafstb.de/ application/DAfStb-Richtlinie_Anforderungen_Ausgangs stoffe_Beton_2019-08-15.pdf

[3] Westendarp, A.; Kunz, C. (2020) Massige Betonbauteile von Wasserbauwerken im (Klima-)Wandel. Beton- und Stahlbeton 115, Sonderheft 100 Jahre MPA Karlsruhe, S. 14-22. https://doi.org/10.1002/best.202000064

[4] DAfStb-Richtlinie Betonbauqualität als Vorlage für DIN 1045:2020, Teil 0 „Grundlagen und Betonbauqualitätsklassen (BBQ)“, Entwurf vom 2. Juni 2020.

[5] DAfStb-Richtlinie Betonbauqualität als Vorlage für DIN 1045:2020, Teil 1 „Bemessung und Konstruktion“, Entwurf vom 21. Februar 2020.

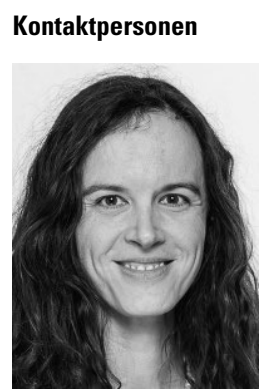

Dr.-Ing. Katja Voland

(Korrespondenzkontaktperson)

voland@betonverein.de

Deutscher Beton- und Bautechnik-Verein E.V.

Kurfürstenstraße 129

10785 Berlin

[6] DAfStb-Richtlinie Betonbauqualität als Vorlage für DIN 1045:2020, Teil 2 „Beton“, Entwurf vom 3. Juni 2020.

[7] DAfStb-Richtlinie Betonbauqualität als Vorlage für DIN 1045:2020, Teil 3 „Bauausführung“, Entwurf vom 20. April 2020.

[8] Kommission „Wachstum, Strukturwandel und Beschäftigung“: Abschlussbericht, Bundesministerium für Wirtschaft und Energie, Druck- und Verlagshaus Zarbock GmbH \& Co.KG, Januar 2019.

[9] Quelle: Verband kommunaler Unternehmen e.V. (VKU) www.vku.de

[10] Feuerborn, H. J.; Kaczmarek, Th. (2019) Ressourcenverfügbarkeit - Konsequenzen für das Bauen mit Beton in der Zukunft - aus der Sicht der Zusatzstoffe: Steinkohlenflugasche. Vortrag auf DAfStb-Fachkolloquium I.

[11] Studie Die Nachfrage nach Primär- und Sekundärrohstoffen der Steine-und-Erden-Industrie bis 2035 in Deutschland, Bundesverband Baustoffe - Steine und Erden e.V., 2019 mit Verweis auf VGB PowerTech e.V.

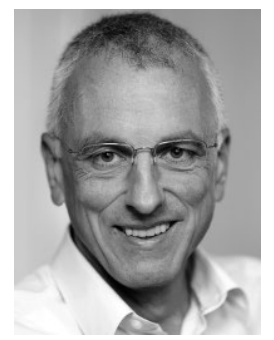

Dipl.-Ing. Andreas Westendarp andreas.westendarp@baw.de Bundesanstalt für Wasserbau Kußmaulstraße 17 76187 Karlsruhe

\section{Zitieren Sie diesen Beitrag}

Voland, K.; Westendarp, A. (2021) Positionspapier „Beton ohne Steinkohlenflugasche“. Beton- und Stahlbetonbau 116, H. 4, S. 322-326. https://doi.org/10.1002/best.202100014 